\title{
Triticum Aestivum L. cv. Guizi 1 ANS-6D Positively Regulates Leaf Senescence Through the Abscisic Acid Mediated Chlorophyll Degradation in Tobacco
}

Luhua Li

Guizhou University

Chang An

Guizhou University

Zhongni Wang

Guizhou Academy of Agricultural Sciences

Fumin Xiong

Guizhou University

Yingxi Wang

Guizhou University

Mingjian Ren

Guizhou University

Ruhong Xu ( $\sim$ xrhgz@163.com )

Guizhou University

\section{Research Article}

Keywords: GzANS-6D, anthocyanin, chlorophyll degradation, ABA, ABF

Posted Date: December 16th, 2021

DOl: https://doi.org/10.21203/rs.3.rs-995236/v1

License: (c) (1) This work is licensed under a Creative Commons Attribution 4.0 International License.

Read Full License 


\section{Abstract}

Anthocyanidin synthase (ANS) is involved in the synthesis of anthocyanins, which are important phytonutrients because of their beneficial effects on human health. Here, we identified ANS-6D of purplecolored Triticum aestivum L. cv. Guizi 1 (Gz) that is involved in leaf senescence through the abscisic acid (ABA) mediated chlorophyll degradation pathway in tobacco. After characterizing the leaf-senescence phenotype in GzANS-6D overexpression (OxGzANS-6D) lines, we found that the increased anthocyanin accumulation and decreased chlorophyll content in $0 x G z A N S-6 D$ lines were closely correlated with the expression levels of anthocyanin synthesis-related structural genes and senescence marker genes, as well as the accumulation of reactive oxygen species. The endogenous ABA content increased and ethylene content decreased in $O x G z A N S-6 D$ transgenic lines compared with wild type. Additionally, the levels of the abscisic acid-responsive transcription factors $A B F 1$ and $A B F 2$, as well as those of chlorophyll degradation-related genes ( $P A O, N Y C, S G R$ and $C H L$ ), were significantly higher in OxGzANS-6D transgenic lines than in wild type. Furthermore, we found that GzABF1 and NtABF1 binds to the promoter of GzANS-6D, and NtABF2 binds to the promoter of NtSGR. Thus, GzANS-6D participated in leaf senescence through ABA-mediated chlorophyll degradation, and $A B F 1 / 2$ play important role in GzANS-6D functions.

\section{Introduction}

Anthocyanins, which are responsible for the colors (e.g., purple, red and blue) of cereal grains, are watersoluble pigments belonging to the flavonoid group (Chen et al. 2013). Purple-colored wheat (Triticum aestivum L.) has purple-colored grains that contain significant levels of anthocyanins (Zeven 1991; Liu et al. 2010). The anthocyanins benefit human health by improving vision and neurological health, and having anticarcinogenic and anti-inflammatory roles owing to their antioxidant activities (Cavalcanti et al. 2011; Khoo et al. 2017). The anthocyanin content is an important indicator of phytonutrients (Wang et al. 2019). Therefore, researches have focused on analyzing anthocyanin synthesis in, and the breeding of, purple-colored wheat.

Structural genes control anthocyanin accumulation through the regulation of the anthocyanin biosynthetic pathway. Phenylalanine ammonia-lyase $(P A L)$, 4-coumarate-CoA ligase (4CL), chalcone synthase $(\mathrm{CHS})$, chalcone isomerase $(\mathrm{CH})$, flavanone 3-hydroxylase $(F 3 H)$, dihydroflavonol 4-reductase $(D F R)$ and anthocyanidin synthase (ANS) are important structural genes in the anthocyanin biosynthetic pathway, among which the ANSencoded anthocyanidin synthase enzyme catalyzes the conversion of leucoanthocyanidin to anthocyanidin (Gonzalez et al. 2008; Jaakola et al. 2013; Li et al. 2018). Transcriptome analyses of genes involved in anthocyanin biosynthesis have demonstrated that ANS plays important roles in anthocyanin biosynthesis in cherry (Prunus avium L.), mulberry (Morus alba L.) and wheat (Wei et al. 2015; Huang et al. 2020; Li et al. 2018). However, only limited studies on ANS functions have demonstrated that overexpressing ANS enhances anthocyanin accumulation, leading to an increased antioxidant potential in rice (Reddy et al. 2007) and reduced salvianolic acid B (SAB) and rosmarinic acid (RA) biosynthesis in Salvia miltiorrhiza and S. miltiorrhiza Bge f. alba (Li et al. 2019) 
plantlets, respectively. Additionally, Fedia graciliflora the ANS knockdown mutants develop larger flowers with wider corolla tube openings than the wild type (WT) (Berger et al. 2017).

Phytohormones play important roles in regulating the anthocyanin content. Both abscisic acid (ABA) and ethylene regulate anthocyanin metabolism (Peng et al. 2011). Early in 1992, Hattor et al. demonstrated that $A B A$ activates the $C 1$ regulatory gene for anthocyanin biosynthesis during seed maturation in maize (Hattor et al. 1992). In addition, ABA plays positive roles in inducing anthocyanin accumulation by regulating the expression of structural and/or regulatory genes in rice (Oryza sativa L.) (Hung and Kao 2005; Hung et al. 2008) and Arabidopsis thaliana (Zhou et al. 2009; Watanabe et al. 2018). The MYB96 mutant (myb96-1d) shows obvious anthocyanin accumulations in leaves after simultaneous stimulation with both ABA and salicylic acid (SA) in Arabidopsis (Seo and Park 2010). González-Villagra et al. (2017) further indicated that ABA participates in anthocyanin synthesis through the regulation of microRNA156, augmenting the expression levels of anthocyanin synthesis-related genes (such as DFR and ANS), resulting in anthocyanin accumulation. Recently, overexpressing the More Axillary Branches 2 gene from Sapium sebiferum in Arabidopsis resulted in higher expression levels of ABA biosynthetic genes, increased anthocyanin accumulations and a higher retained chlorophyll content (Wang et al. 2019). Interestingly, the ABA content is elevated along with anthocyanin accumulation in the OsPL6 (Purple Leaf) mutant; however, the chlorophyll content is significantly reduced at the tillering stage (Khan et al. 2020). Additionally, the ABA and anthocyanin contents increase, while the chlorophyll content decreases in torenia (Torenia fournieri) and allotetraploid rapeseed (Brassica napus L.) under sucrose and severe salinity treatments, respectively (Nagira et al. 2006; Feng et al. 2020). Ethylene signaling plays a negative role in Pi starvation- (Lei et al. 2011), sucrose- (Jeong et al. 2010; Kwon et al. 2011; Meng et al. 2018) and light-induced (Jeong et al. 2010) anthocyanin accumulation in Arabidopsis. Sewelam et al. demonstrated that ETHYLENE RESPONSE FACTOR6 insertion mutant plants showed increased $\mathrm{H}_{2} \mathrm{O}_{2}$ and anthocyanin levels in Arabidopsis (Sewelam et al. 2013). Under nitrogen starvation conditions, the ROOT HAIR DEFECTIVE3 mutant shows an anthocyanin overaccumulation phenotype similar to ethylene signaling mutants etr1, ein2 and ein3/eil1 in Arabidopsis. Additionally, the ethylene precursor ACC strongly suppresses anthocyanin accumulation in a partially ROOT HAIR DEFECTIVE3-dependent manner (Wang et al. 2015). Recently, ethylene was reported to repress anthocyanin biosynthesis and accumulation in pericarps in the dark, and the aminoethoxyvinylglycine hydrochloride (an ethylene biosynthesis inhibitor) treatment results in significantly higher transcript levels of biosynthetic genes (e.g., DFR, CHS and ANS) in rice (Kumar et al. 2019). Carvalho et al. (2010) reported that ABA and anthocyanin biosynthetic genes in the serine/arginine-rich 45 mutant were over-induced by glucose; however, there is no effect on ethylene signaling (Carvalho et al. 2010). Most reports have shown that ABA and ethylene participate in regulation anthocyanin biosynthesis in Arabidopsis and rice, however, the effects of ABA on anthocyanin biosynthesis in wheat still require research.

Based on the transcriptome sequencing results (Li et al. 2018), we found that the expression levels of the structural GzANSs (especially GzANS-6D) underwent positive dynamic changes along with anthocyanin accumulation. Here, we characterized the functions of GzANS-6D in tobacco. We first analyzed the 
dynamic changes in GzANS-6D in three important developmental periods of 'Guizi 1' (Gz) wheat. Then, we analyzed the phenotypes of transgenic tobacco lines overexpressing GzANS-6D (OxGzANS-6D) grown under normal conditions. The $O x G z A N S-6 D$ lines showed early senescence phenotypes with higher anthocyanin contents and lower chlorophyll contents than WT. The OxGzANS-6D lines had higher anthocyanin biosynthetic and senescence-related gene expression levels than the WT. Consistently, these transgenic lines displayed accumulations of reactive oxygen species (ROS) content and losses in antioxidant enzyme activity levels. Furthermore, the endogenous contents of $A B A$ and ethylene were detected, and the $O x G z A N S-6 D$ lines possessed higher ABA content and lower ethylene content compare with WT. Finally, compared with the WT plants, the OxGzANS-6D lines exhibited higher expression levels of $A B F s$ and chlorophyll degradation-related genes. These results demonstrated that GzANS-6D participated in leaf senescence through the regulation of endogenous $A B A$ signaling.

\section{Materials And Methods}

\section{Plant materials and plant growth conditions}

Triticum aestivum L. cv. Guizi 1 (Certificate No. Qian2015003) was planted on the experimental farm, and the field management was performed in accordance with Li et al. (Li et al. 2018). The developing caryopses were selected from spikes at the identical flowering stages at 10-, 15- and 25-days post anthesis (dpa), and samples were stored at $-80^{\circ} \mathrm{C}$ for RNA extraction. The coding sequence of GzANS$6 D$ was obtained from 25 dpa caryopses of 'GZ1', placed into the pBI121 vector, and then transformed into the tobacco (Nicotiana benthamiana). From the regenerated plants, transgenic GzANS-6D(OxGzANS$6 D$ ) overexpression lines were selected (Luo et al. 2006). The OxGzANS-6D transgenic lines were confirmed using PCR. Seeds were surface sterilized with 75\% [v/v] ethanol for 2 min and then 1\% [v/v] sodium hypochlorite for $10 \mathrm{~min}$, followed by washing four times for $5 \mathrm{~min}$ each time with distilled water. Seeds were vernalized at $4^{\circ} \mathrm{C}$ for 3 days in the dark. Seedlings were grown vertically on MS medium. Twoweek-old plants were transferred to soil. The plants were grown at $25^{\circ} \mathrm{C} / 22^{\circ} \mathrm{C}$ (day/night) under long-day conditions (16-h light/8-h dark). The 7th and/or 8th leaves (cotyledons were excluded) were harvested from 10-week-old tobacco plants for RNA extraction, and the determination of anthocyanin, chlorophyll and phytohormone contents, ROS accumulation levels and enzyme activities.

\section{RNA isolation and quantitative real-time PCR}

Total RNAs from wheat and tobacco were extracted using an EASYspin Plus Complex Plant RNA Kit (Aidlab, Beijing, China), and then, first-strand cDNA was synthesized from $1 \mu \mathrm{g}$ of RNA using a TUREscript 1st Strand cDNA Synthesis Kit gDNA Eraser (Aidlab, Beijing, China). The $\beta$-actin gene was used as the internal control, and a qRT-PCR analysis was performed using LightCycler480 SYBR Green (Bio-Rad, CFX 96 Touch, USA). The primers for qRT-PCR are listed in Table S1.

\section{Extraction and measurement of total anthocyanins}


The extraction and total content determination of anthocyanins were performed in accordance with Li et al. (Li et al. 2018). Briefly, $0.5 \mathrm{~g}$ frozen samples were ground into powder in liquid nitrogen, then $4 \mathrm{~mL}$ of acidified ethanol was added and mixed with each sample for anthocyanin extraction. The total anthocyanins level was determined by measuring the absorbance at 527 with an Evolution 220 (Thermo Fisher, USA) and calculated using the following formula: anthocyanin content $(\mathrm{mg} / \mathrm{Kg})=(C \times V) / m$, where $C$ represents anthocyanin concentration, $V$ represents volume and $m$ represents sample weight).

\section{Extraction and determination of total chlorophyll}

The extraction and determination of chlorophyll were performed in accordance with previously described protocols (Zhang and Guo 2018; Kong et al. 2014). Briefly, $0.25 \mathrm{~g}$ fresh leaf samples were used for chlorophyll extraction, and $12.5 \mathrm{~mL}$ extraction solution was added at room temperature in darkness until the leaf tissue was completely bleached. The leaves were incubated in the dark for $24 \mathrm{~h}$ at room temperature. The absorbance levels of the solution were measured at 665 and $649 \mathrm{~nm}$ using an Evolution 220 (Thermo Fisher) and calculated using the following formulae: Chlorophyll a content $(\mathrm{Ca})(\mu \mathrm{g} / \mathrm{mL})=$ 13.95×A665-6.88×A649; Chlorophyll b content $(\mathrm{Cb})(\mu \mathrm{g} / \mathrm{mL})=24.96 \times A 649-7.32 \times A 665 ;$ and Total chlorophyll $(\mathrm{mg} / \mathrm{g})=(C a+C b) \times V / W$, where $V$ represents the volume of the extraction solution; $W$ represents the weight of freshly detached leaves.

\section{Histochemical staining of ROS and an antioxidant enzyme activity analysis}

$\mathrm{H}_{2} \mathrm{O}_{2}$ and $\mathrm{O}_{2} .^{-}$were detected using a 3,3'-diaminobenzidine (DAB) and nitroblue tetrazolium chloride (NBT) staining kit (Solarbio, Beijing, China). Then, the leaves were imaged and used to calculate the relative gray value with ImageJ software (black is 0 and white is 255). The activity levels of antioxidant enzymes (POD, CAT and SOD) were detected using the appropriate corresponding kit (Suzhou Grace Biotechnology Co.Ltd).

\section{Determination of phytohormones}

Endogenous ABA levels were measured using a plant ELISA kit (Shanghai Enzyme-linked Biotechnology Co. Ltd., Shanghai, China) following the producer's instructions. Leaves of $O x G z A N S-6 D$ transgenic plants and WT were ground in liquid nitrogen and used to measure the ABA content. The calibration standards and samples were simultaneously determined by measuring the absorbance at $450 \mathrm{~nm}$ using a microplate reader (Infinite F50, TECAN). The standard curve was constructed using the concentrations of calibration standards against optical density (OD). Then, the sample ABA contents were established by tracking the standard curve and establishing similar trends using previously reported sample values (Khan et al., 2020). The endogenous ethylene content was measured in accordance with Li et al. (2014). Briefly, $0.2 \mathrm{~g}$ of $O x G z A N S-6 D$ transgenic plants and WT leaves were placed separately in 1-mL gas-tight glass vessels. A drop of distilled water was added, the caps tightened and the samples incubated at room temperature for $24 \mathrm{~h}$. For ethylene determination, $5 \mu \mathrm{L}$ sample of gas was removed and analyzed using a gas chromatograph (GC7890, Agilent, USA) with a chromatographic column (hp-5; $30 \times 0.25 \mathrm{~mm}$ ). The oven, injector and detector temperatures were 50,150 and $230^{\circ} \mathrm{C}$, respectively. 


\section{Yeast one-hybrid $(\mathrm{Y} 1 \mathrm{H})$ assay}

The $\mathrm{Y} 1 \mathrm{H}$ was performed using the Matchmaker Gold Yeast One-Hybrid Library Screening System (Clontech). The cDNA sequence was cloned into the prey vector pGADT7 and transformed into the yeast strain $\mathrm{Y} 1 \mathrm{H}$ Gold. The GzANS-6D and NtSGR promoter was inserted into the pABAi-bait vector.

\section{Results}

\section{Phylogenetic tree, multiple alignment and expression levels of GzANS genes}

Based on the transcriptome sequencing results of three important periods ( $10 \mathrm{dpa}, 25 \mathrm{dpa}$ and $35 \mathrm{dpa}$ ) of 'Guizi 1' for anthocyanin biosynthesis, we found that the structural GzANS genes' expression levels positively changed along with anthocyanin accumulation (Fig. 1A). The expression levels of GzANSS were significantly higher at $25 \mathrm{dpa}$ and $35 \mathrm{dpa}$ than at $10 \mathrm{dpa}$. They exhibited dynamic increases from 10 to $25 \mathrm{dpa}$ and then decreased from 25 to $35 \mathrm{dpa}$. Among the GzANSs, GzANS-6D exhibited a remarkable change. Then, qRTPCR validation was carried out using GzANS-6D in 'Guizi 1' and 'Chinese Spring' wheat (Fig. 1B). In the former, GzANS-6D was significantly higher at $25 \mathrm{dpa}$ and $35 \mathrm{dpa}$ than at $10 \mathrm{dpa}$. It exhibited a dynamic increase from 10 to $25 \mathrm{dpa}$ and then decreased from 25 to $35 \mathrm{dpa}$, which coincided with the transcriptome sequencing results. However, in the latter, there were no significant differences in GzANS-6D expression at the different developmental periods. A phylogenetic tree analysis was carried out for ANS proteins having protein homology levels greater than 70\% compared with GzANS-6D (Fig. S1). The ANSs clustered into several subgroups, with GzANSs and TaANSs being in the same group. Multiple alignments of ANSs in wheat revealed that the protein sequences of GzANS-6D and GzANS-6A1 were identical to those of TaANS-D1 and TaANS-A1, respectively, and the protein sequences of GzANS-6B were different by one amino acid from TaANS-B1 (Fig. S2). The ANSs are involved in the regulation of anthocyanin biosynthesis.

\section{GzANS-6D overexpression promotes anthocyanin accumulation and leaf senescence in tobacco}

To further understand physiological functions of GzANS-6D in plant growth and development, we generated GzANS-6D-overexpression (OxGzANS-6D) lines in tobacco. The rosette diameters and the seventh-leaf sizes (including the length and width) of 5-week-old seedlings of OxGzANS-6D lines were significantly smaller than those of the WT (Fig. S3). OxGzANS-6D lines exhibited an early senescence phenotype in 10-week-old transgenic plants (Fig. 2A). Subsequently, the anthocyanin and chlorophyll contents of the seventh leaves were detected. The former was significantly higher in OxGzANS-6D lines than in WT (Fig. 2B); however, the latter was significantly lower in OxGzANS-6D lines than in WT (Fig. 2C).

Our laboratory previously determined that anthocyanin accumulation is often accompanied by the upregulated expression of anthocyanin synthesis-related structural genes (Li et al. 2018). In our study, RNA was extracted from the seventh leaves, and the transcript levels of six structural genes, PAL, 4CL, CHS, $C H I, F 3 H$ and $D F R$, were detected using qPCR. The transcript levels of these genes were significantly higher in OxGzANS-6D transgenic leaves than those in WT leaves (Fig. 3A-F). The senescence marker 
genes glutamate dehydrogenase (GDH1), din, cysteine protease (CP1) and senescence-associated gene (SAG12) are upregulated during leaf senescence (Li et al., 2016; Liu et al., 2016). Here, the transcript levels of these four genes were significantly higher in $0 x G z A N S-6 D$ transgenic leaves than in WT leaves (Fig. 4A-D), with the transcript levels of $C P 1$ and $S A G 12$ being increased by more than 40 -fold (Fig. 4C) and 90-fold (Fig. 4D) in OXGzANS-6D transgenic leaves compared with WT leaves, respectively. Thus, the overexpression of GzANS-6D, which results in anthocyanin accumulation, correlates with plant senescence. Furthermore, the yield traits of the WT and $O x G z A N S-6 D$ transgenic lines revealed that the latter had significantly lower caryopsis numbers and thousand grain weights than the WT (Table 1), which coincided with their early senescence phenotype.

Table 1

Analysis of yield traits in WT and transgenic lines

\begin{tabular}{|lllll|}
\hline Yield traits & WT & OE6 & OE7 & OE9 \\
\hline Caryopsis number & $43.00 \pm 1.41$ & $33.75 \pm 1.71^{\star \star}$ & $34.00 \pm 1.83^{\star \star}$ & $32.25 \pm 2.87^{\star \star}$ \\
\hline Thousand grains weight $(\mathrm{mg})$ & $65.33 \pm 1.49$ & $58.67 \pm 1.70^{\star \star}$ & $60.67 \pm 1.11^{\star \star}$ & $59.33 \pm 1.11^{\star \star}$ \\
\hline Note: ${ }^{\star \star *}$ means extremely significant level $(\mathrm{P}<0.01)$. & & \\
\hline
\end{tabular}

\section{Overexpression of GzANS-6D increases ROS accumulation and reduces antioxidant enzyme activity levels}

To verify the function of GzANS-6D in leaf senescence, we performed assays to measure the accumulation of ROS $\left(\mathrm{H}_{2} \mathrm{O}_{2}\right.$ and $\left.\mathrm{O}_{2}{ }^{-}\right)$in the leaves of the WT and $O x G z A N S-6 D$ plants. The eighth fresh leaves of 10-week-old plants were used for DAB and NBT staining to detect the accumulations of $\mathrm{H}_{2} \mathrm{O}_{2}$

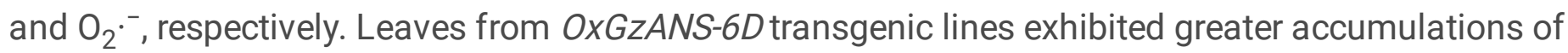
$\mathrm{H}_{2} \mathrm{O}_{2}$ (Fig. 5A and 5C) and $\mathrm{O}_{2}{ }^{-}$(Fig. 5B and 5D) than leaves from the WT. ROS levels in plants are controlled by biosynthetic processes and antioxidant systems (Mittler et al. 2004). Therefore, we detected the activity levels of the antioxidant enzymes POD (Fig. 6A), CAT (Fig. 6B) and SOD (Fig. 6C). All three antioxidant enzymes' activity levels were significantly decreased in OxGzANS-6D transgenic lines compared with WT. These results indicated that GzANS-6D participates in leaf senescence by regulating ROS accumulation-associated biological processes.

\section{Endogenous ABA and ethylene content changes in 0xGzANS-6D transgenic plants}

The ABA and ethylene contents were determined in the leaves of WT and OXGzANS-6D plants. The seventh fresh leaves of 10-week-old plants were used to analyze endogenous $A B A$ and ethylene contents. The ABA content significantly increased in $0 x G z A N S-6 D$ transgenic lines compared with WT (Fig. 7A); however, the ethylene content significantly decreased in $0 x G z A N S-6 D$ transgenic lines compared with WT (Fig. 7B). Thus, ABA and ethylene may play different roles during anthocyanin accumulation in OxGzANS$6 D$ plants. 
ABFs and chlorophyll degradation-related genes are upregulated in 0xGzANS-6D transgenic plants

To further investigate the role of $A B A$ in the leaf senescence of $O x G z A N S-6 D$ transgenic lines, we performed an RT-qPCR analysis on ABA-responsive element (ABRE)-binding transcription factors genes, such as $A B F 1$ and $A B F 2$, and several chlorophyll degradation-related genes, such as pheophorbide a oxygenase $(P A O)$, non-yellow coloring $(N Y C)$, non-yellowing (SGR, also known as $N Y E$ ) and chlorophyllase ( $C H L$ ) (Fig. 8). The ABFs are direct activators of $P A O, N Y C$ and $S G R$, which are involved in the regulation of chlorophyll degradation (Gao et al. 2016; Hu et al. 2019). The expression levels of $A B F 1$ (Fig. 8A) and $A B F 2$ (Fig. 8B) were significantly higher in $O x G z A N S-6 D$ transgenic plants than in WT. In addition, the expression levels of four chlorophyll degradation-related genes (PAO,NYC, SGR and $C H L)$ were significantly higher in OxGzANS-6D transgenic lines compared with WT (Fig. 8C-F).

\section{GzABF1 and NtABF1 binds to the promoter of GzANS-6D, and NtABF2 binds to the promoter of NtSGR}

As indicated in Fig. 9A and 9B, a ABRE sequence of GzANS-6D promoter was identified as the possible binding region by ABFs (Fig. 9A). Y1H assay indicated GzABF1 and NtABF1 binds to the GzANS-6D promoter, whereas NtABF2 cannot binds to the promoter of GzANS-6D (Fig. 9B). Meanwhile, $\mathrm{Y} 1 \mathrm{H}$ assay indicated that NtABF2 binds to the NtSGR protomer (Fig. 9C). The results demonstrated the expression of GzANS-6D regulated by GzABF1 and NtABF1, and expression of NtSGR regulated by NtABF2 (Fig. 9).

\section{Discussion}

Transcriptome analyses of genes involved in anthocyanin biosynthesis have demonstrated the importance of ANS (Zhang et al. 2016; Li et al. 2018; Huang et al. 2020). Additionally, limited studies demonstrated that ANS plays important roles in antioxidant potential, phenolic acids biosynthesis and floral morphology (Reddy et al. 2007; Li et al. 2019; Berger et al. 2017). Therefore, more studies are needed to explore functions of ANS. Here, our transcriptome sequencing (Fig. 1A) and qRTPCR validation (Fig. 1B) results demonstrated that GzANS-6D positively changed with anthocyanin accumulation in wheat, which is in agreement with our previous study (Li et al. 2018).

To further understand the functions of GzANS-6D, we explored the phenotypes of $0 x G z A N S-6 D$ transgenic lines under normal growth conditions. We found that the transgenic lines had smaller rosette diameters (Figure S3) and leaf senescence phenotypes (Fig. 2A) compared with WT. The transgenic lines had higher anthocyanin contents (Fig. 2B) and lower chlorophyll contents (Fig. 2C) compared with WT, which corresponded to GzANS-6D's function and leaf senescence phenotype, respectively. This result coincides with the functions of LcABFs (Hu et al. 2019) and OsPL6 (Khan et al. 2020), which demonstrated that anthocyanin and chlorophyll have antagonistic effects. Anthocyanin accumulation is often accompanied by the up-regulated expression of anthocyanin synthesis-related structural genes (Li et al. 2018). Out data also show that six structural genes ( $P A L, 4 C L, C H S, C H I, F 3 H$ and $D F R)$ involved in anthocyanin synthesis were upregulated in OxGzANS-6D transgenic lines compared with WT (Fig. 3). Leaf senescence is often accompanied by the up-regulated expression of senescence marker genes. Consequently, four senescence marker genes ( $G D H 1$, din, CP1 and SAG12) were selected based on the previous reports (Li et 
al. 2016; Liu et al. 2016) and their expression levels were determined. qRT-PCR confirmed that these four genes were upregulated in OXGzANS-6D transgenic lines compared with in WT (Fig. 4), which was in agreement with the premature senescence characteristic. Additionally, the early leaf senescence of OxGzANS-6D transgenic lines resulted in lower yields compared with WT, which was consistent with the phenotype of small auxin-up RNA 39 overexpression in rice (Kant et al. 2009). Leaf senescence is often accompanied by ROS accumulation (Cui et al. 2013; Liu et al. 2016; Zhang et al. 2020). Our DAB (for $\mathrm{H}_{2} \mathrm{O}_{2}$ content) and NBT (for $\mathrm{O}_{2} .^{-}$content) staining results indicated that GzANS-6D positively regulated ROS accumulation (Fig. 5). Antioxidant proteins (such as POD, CAT and SOD) act as ROS-scavenging antioxidants by reducing the ROS level (Nakabayashi et al., 2014; Lotkowska et al., 2015). Here, all three antioxidant enzymes' activity levels decreased in OxGzANS-6D transgenic lines compared with WT (Fig. 6), which indicated that GzANS-6D participates in leaf senescence by regulating ROS signaling.

ABA promotes, but ethylene inhibits, the accumulation of anthocyanin (Peng et al. 2011) and ROS (Yu et al. 2019). Here, we demonstrated that the endogenous ABA content significantly increased and the ethylene content significantly decreased in OxGzANS-6D transgenic lines compared with WT (Fig. 7), which was in agreement with previous studies (Peng et al. 2011). Therefore, we hypothesized that endogenous $A B A$ and ethylene play different roles in the leaf senescence of $O X G z A N S-6 D$ transgenic lines. In Arabidopsis, ABF2/3/4 were identified as direct activators of NYE1/2, NYC1 and PAO, which participate in chlorophyll degradation during leaf senescence processes in an ABA-dependent manner (Gao et al. 2016). Recently, in Litchi chinensis, ABF1/2 were identified as recognizing ABA-responsive elements in the promoter regions of $P A O$ and $S G R$, and they are, therefore, involved in chlorophyll degradation (Hu et al. 2019). qRT-PCR confirmed that the expression levels of ABF1/2 and four chlorophyll degradation-related genes ( $P A O, N Y C$, SGR and $C H L$ ) were significantly higher in OxGZANS-6D transgenic lines than in WT (Fig. 8). Furthermore, we found GzABF1 and NtABF1 can directly binds to the promoter of GzANS-6D, whereas NtABF1 cannot. Meanwhile, NtABF2 binds to the promoter of NtSGR (Fig. 9). Our results strongly suggest that GzANS-6D participates in leaf senescence through in an ABAmediated chlorophyll-degradation manner.

Our results demonstrated that $G z A N S-6 D$ positively regulates anthocyanin synthesis and leaf senescence by increasing the endogenous $A B A$ content and decreasing the ethylene content. ABA-mediated chlorophyll degradation through ABF2's activation of chlorophyll degradation-related gene (including $P A O, N Y C, S G R$ and $C H L$, mainly $S G R$ ) expression in tobacco. In addition, ABA play important regulatory roles in ROS signaling in leaf senescence. Our work reveals a novel regulatory module containing $A N S$, and $A B A$ that is involved in leaf senescence.

\section{Conclusions}

Overexpression of GzANS-6D promote leaf senescence through regulating the ABA-mediated chlorophyll degradation pathway, and $A B F 1 / 2$ play important role in GzANS-6D functions.

\section{Declarations}


Conflict of Interest

The authors declare that the research was conducted in the absence of any commercial or financial relationships that could be construed as a potential conflict of interest.

Acknowledgments

This project was supported by grants from the National Natural Science Foundation of China (3216150357), Guizhou Science and technology support project ([2021] YiBan272), Guizhou Science and Technology Plan Project ([2020] 1Z018), the Guizhou Science and Technology Plan Project ([2019] 1073) and Achievement's promotion project by Department of Science and Technology of Guizhou Province ([2019] 4246). We thank International Science Editing ( http://www.internationalscienceediting.com ) for editing this manuscript.

\section{References}

Berger BA, Ricigliano VA, Savriama Y, Lim A, Thompson V, Howarth DG (2017) Geometric morphometrics reveals shifts in flower shape symmetry and size following gene knockdown of CYCLOIDEA and ANTHOCYANIDIN SYNTHASE. BMC Plant Biol 17:205. https://doi.org/10.1186/s12870-017-1152-x.

Carvalho RF, Carvalho SD, Duque P (2010) The plant-specific SR45 protein negatively regulates glucose and ABA signaling during early seedling development in Arabidopsis. Plant Physiol 154:772783. https://doi.org/10.1104/pp.110.155523. Epub 2010 Aug 10.

Cavalcanti RN, Santos DT, Meireles MAA (2011) Non-thermal stabilization mechanisms of anthocyanins in model and food systems--an overview. Food Res Int 44:499509. https://doi.org/10.1016/j.foodres.2010.12.007.

Chen W, Müller D, Richling E, Wink M (2013) Anthocyanin-rich purple wheat prolongs the life span of Caenorhabditis elegans probably by activating the DAF-16/FOXO transcription factor. J Agric Food Chem 61:3047-3053. https://doi.org/10.1021/jf3054643. Epub 2013 Mar 18.

Cui MH, Ok SH, Yoo KS, Jung KW, Yoo SD, Shin JS (2013) An Arabidopsis cell growth defect factor-related protein, CRS, promotes plant senescence by increasing the production of hydrogen peroxide. Plant Cell Physiol 54:155-167. https://doi.org/10.1093/pcp/pcs161. Epub 2012 Dec 4.

Feng YN, Cui JQ, Zhou T, Liu Y, Yue CP, Huang JY, Hua YP (2020) Comprehensive dissection into morphophysiologic responses, ionomic homeostasis, and transcriptomic profiling reveals the systematic resistance of allotetraploid rapeseed to salinity. BMC Plant Biol 20:534. https://doi.org/10.1186/s12870020-02734-4.

Gao S, Gao J, Zhu XY, Song Y, Li ZP, Ren GD, Zhou X, Kuai BK (2016) ABF2, ABF3 and ABF4 promote ABAmediated chlorophyll degradation and leaf senescence by transcriptional activation of chlorophyll 
catabolic genes and senescence-associated genes in Arabidopsis. Mol Plant 9:12721285. https://doi.org/10.1016/j.molp.2016.06.006. Epub 2016 Jun 30.

Gonzalez A, Zhao M, Leavitt JM, Lloyd AM (2008) Regulation of the anthocyanin biosynthetic pathway by the TTG1/bHLH/Myb transcriptional complex in Arabidopsis seedlings. Plant J 53:814827. https://doi.org/10.1111/j.1365-313X.2007.03373.x. Epub 2007 Nov 23.

González-Villagra J, Kurepin LV, Reyes-Díaz MM (2017) Evaluating the involvement and interaction of abscisic acid and miRNA156 in the induction of anthocyanin biosynthesis in drought-stressed plants. Planta 246:299-312. https://doi.org/10.1007/s00425-017-2711-y. Epub 2017 May 22.

Hu B, Lai B, Wang D, Li JQ, Chen LH, Qin YQ, Wang HC, Qin YH, Hu GB, Zhao JT (2019) Three LcABFs are involved in the regulation of chlorophyll degradation and anthocyanin biosynthesis during fruit ripening in Litchi chinensis. Plant Cell Physiol 60:448-461. https://doi.org/10.1093/pcp/pcy219.

Hung KT, Kao CH (2005) Hydrogen peroxide is necessary for abscisic acid-induced $\mathrm{NH}^{4+}$ accumulation in rice leaves. J Plant Physiol 162:1022-1029. https://doi.org/10.1016/j.jplph.2004.11.007.

Hung KT, Cheng DG, Hsu YT, Kao CH (2008) Abscisic acid-induced hydrogen peroxide is required for anthocyanin accumulation in leaves of rice seedlings. J Plant Physiol 165:1280-1287. https://doi.org/10.1016/j.jplph.2007.10.008. Epub 2007 Dec 26.

Huang GQ, Zeng YC, Wei L, Yao YQ, Dai J, Liu G, Gui ZZ (2020) Comparative transcriptome analysis of mulberry reveals anthocyanin biosynthesis mechanisms in black (Morus atropurpurea Roxb.) and white (Morus alba L.) fruit genotype. BMC Plant Biol 20:279. https://doi.org/10.1186/s12870-020-02486-1.

Jaakola $L$ (2013) New insights into the regulation of anthocyanin biosynthesis in fruits. Trends Plant Sci 18:477-483. https://doi.org/10.1016/j.tplants.2013.06.003.

Jeong SW, Das PK, Jeoung SC, Song JY, Lee HK, Kim YK, Kim WJ, Park YI, Yoo SD, Choi SB, Choi G, Park YI (2010) Ethylene suppression of sugar-induced anthocyanin pigmentation in Arabidopsis. Plant Physiol 154:1514-1531. https://doi.org/10.1104/pp.110.161869. Epub 2010 Sep 27.

Kant S, Bi YM, Zhu T, Rothstein SJ (2009) SAUR39, a small auxin-up RNA gene, acts as a negative regulator of auxin synthesis and transport in rice. Plant Physiol 151:691701. https://doi.org/10.1104/pp.109.143875. Epub 2009 Aug 21.

Khan A, Jalil S, Cao H, Tsago Y, Sunusi M, Chen ZY, Shi CH, Jin XL (2020) The purple leaf (p/6) mutation regulates leaf color by altering the anthocyanin and chlorophyll contents in rice. Plants (Basel) 9:1477. https://doi.org/10.3390/plants9111477.

Khoo HE, Azlan A, Tang ST, Lim SM (2017) Anthocyanidins and anthocyanins: colored pigments as food, pharmaceutical ingredients, and the potential health benefits. Food Nutr Res 
Kong J, Dong Y, Xu L, Liu S, Bai X (2014) Effects of foliar application of salicylic acid and nitric oxide in alleviating iron deficiency induced chlorosis of Arachis hypogaea L. Bot Stud 55:9. https://doi.org/10.1186/1999-3110-55-9. Epub 2014 Jan 20.

Kumar D, Sarmah BK, Das PK (2019) Ethylene mediates repression of anthocyanin accumulation in black rice pericarps in the absence of light. J Plant Physiol 236:34-38. https://doi.org/10.1016/j.jplph.2019.02.011. Epub 2019 Feb 26.

Kwon Y, Oh JE, Noh H, Hong SW, Bhoo SH, Lee H (2011) The ethylene signaling pathway has a negative impact on sucrose-induced anthocyanin accumulation in Arabidopsis. J Plant Res 124:193-200. https://doi.org/10.1007/s10265-010-0354-1. Epub 2010 Jun 1.

Lei MG, Zhu CM, Liu YD, Karthikeyan AS, Bressan RA, Raghothama KG, Liu D (2010) Ethylene signalling is involved in regulation of phosphate starvation-induced gene expression and production of acid phosphatases and anthocyanin in Arabidopsis. New Phytol 189:1084-1095.

https://doi.org/10.1111/j.1469-8137.2010.03555.x. Epub 2010 Nov 30.

Li HY, Liu JL, Pei TL, Bai ZQ, Han RL, Liang ZS (2019) Overexpression of SmANS enhances anthocyanin accumulation and alters phenolic acids content in Salvia miltiorrhiza and Salvia miltiorrhiza Bge f. alba plantlets. Int J Mol Sci 20:2225. https://doi.org/10.3390/ijms20092225.

Li JS, Jia HL, Wang J (2014) cGMP and ethylene are involved in maintaining ion homeostasis under salt stress in Arabidopsis roots. Plant Cell Rep 33:447-459. https://doi.org/10.1007/s00299-013-1545-

8. Epub 2013 Dec 4.

Li LH, Xing YF, Chang D, Fang SS, Cui BY, Li Q, Wang XJ, Guo S, Yang X, Men SZ, Shen YQ (2016) CaM/BAG5/Hsc70 signaling complex dynamically regulates leaf senescence. Sci Rep 6:31889. https://doi.org/10.1038/srep31889.

Li XL, Qian XK, Lǔ X, Wang XH, Ji N, Zhang MS, Ren MJ (2018) Upregulated structural and regulatory genes involved in anthocyanin biosynthesis for coloration of purple grains during the middle and late grain-filling stages. Plant Physiol Biochem 130:235-

247. https://doi.org/10.1016/j.plaphy.2018.07.011. Epub 2018 Jul 10.

Liu Q, Qiu Y, Beta T (2010) Comparison of antioxidant activities of different colored wheat grains and analysis of phenolic compounds. J Agric Food Chem 58:9235-9241.

https://doi.org/10.1021/jf101700s. Epub 2010 Jul 29.

Liu Y, Wang L, Liu H, Zhao RR, Liu B, Fu QJ, Zhang YH (2016) The antioxidative defense system is involved in the premature senescence in transgenic tobacco (Nicotiana tabacum NC89). Biol Res 49:30. https://doi.org/10.1186/s40659-016-0088-1. 
Lotkowska ME, Tohge T, Fernie AR, Xue GP, Balazadeh S, MuellerRoeber B (2015) The Arabidopsis transcription factor MYB112 promotes anthocyanin formation during salinity and under high light stress. Plant Physiol 169:1862-1880. https://doi.org/10.1104/pp.15.00605. Epub 2015 Sep 16.

Luo K, Zheng X, Chen Y, Xiao Y, Zhao D, McAvoy R, Pei Y, Li Y (2006) The maize Knotted1 gene is an effective positive selectable marker gene for Agrobacterium-mediated tobacco transformation. Plant Cell Rep 25:403-409. https://doi.org/10.1007/s00299-005-0051-z. Epub 2005 Dec 21.

Meng LS, Xu MK, Wan W, Yu F, Li C, Wang JY, Wei ZQ, Lv MJ, Gao XY, Li ZY, Jiang HJ (2018) Sucrose signaling regulates anthocyanin biosynthesis through a MAPK cascade in Arabidopsis thaliana. Genetics 210:607-619. https://doi.org/10.1534/genetics.118.301470. Epub 2018 Aug 16.

Mittler R, Vanderauwera S, Gollery M, Breusegem FV (2004) Reactive oxygen gene network of plants. Trends Plant Sci 9:490-498. https://doi.org/10.1016/j.tplants.2004.08.009.

Nagira Y, Ikegami K, Koshiba T, Ozeki Y (2006) Effect of ABA upon anthocyanin synthesis in regenerated torenia shoots. J Plant Res 119:137-144. https://doi.org/10.1007/s10265-005-0256-9. Epub 2006 Feb 8.

Nakabayashi R, Yonekura-Sakakibara K, Urano K, Suzuki M, Yamada Y, Nishizawa T (2014) Enhancement of oxidative and drought tolerance in Arabidopsis by overaccumulation of antioxidant flavonoids. Plant $\mathrm{J}$ 77:367-379. https://doi.org/10.1111/tpj.12388. Epub 2013 Dec 17.

Peng Z, Han C, Yuan L, Zhang K, Huang H, Ren C (2011) Brassinosteroid enhances jasmonate-induced antho-cyanin accumulation in Arabidopsis seedlings. J Integr Plant Biol 53:632640. https://doi.org/10.1111/j.1744-7909.2011.01042.x.

Reddy AM, Reddy VS, Scheffler BE, Wienand U, Reddy AR (2007) Novel transgenic rice overexpressing anthocyanidin synthase accumulates a mixture of flavonoids leading to an increased antioxidant potential. Metab Eng 9:95-111. https://doi.org/10.1016/j.ymben.2006.09.003. Epub 2006 Oct 4.

Seo PJ, Park C M (2010) MYB96-mediated abscisic acid signals induce pathogen resistance response by promoting salicylic acid biosynthesis in Arabidopsis. New Phytol 186:471483. https://doi.org/10.1111/j.1469-8137.2010.03183.x. Epub 2010 Feb 8.

Sewelam N, Kazan K, Thomas-Hall SR, Kidd BN, Manners JM, Schenk PM (2013) Ethylene response factor 6 is a regulator of reactive oxygen species signaling in Arabidopsis. PLoS One 8:e70289. https://doi.org/10.1371/journal.pone.0070289. Print 2013.

Wang J, Wang Y, Yang J, Ma CL, Zhang Y, Ge T, Qi Z, Kang Y (2015) Arabidopsis ROOT HAIR $D E F E C T I V E 3$ is involved in nitrogen starvation-induced anthocyanin accumulation. $J$ Integr Plant Biol 57:708-721. https://doi.org/10.1111/jipb.12320. Epub 2015 Feb 2. 
Wang F, Sha JC, Chen Q, Xu XX, Zhu ZL, Ge SF, Jiang YM (2019) Exogenous abscisic acid regulates distribution of ${ }^{13} \mathrm{C}$ and ${ }^{15} \mathrm{~N}$ and anthocyanin synthesis in 'Red Fuji' apple fruit under high nitrogen supply. Front Plant Sci 10:1738. https://doi.org/10.3389/fpls.2019.01738. eCollection 2019.

Wang QJ, Ni J, Shah F, Liu WB, Wang DD, Yao YY, Hu Y, Huang SW, Hou JY, Fu SL, Wu LF (2019) Overexpression of the stress-inducible SsMAX2 promotes drought and salt resistance via the regulation of redox homeostasis in Arabidopsis. Int J Mol Sci 20:837. https://doi.org/10.3390/ijms20040837.

Watanabe S, Sato M, Sawada Y, Tanaka M, Matsui A, Kanno Y, Hirai MY, Seki M, Sakamoto A, Seo M (2018) Arabidopsis molybdenum cofactor sulfurase ABA3 contributes to anthocyanin accumulation and oxidative stress tolerance in ABA dependent and independent ways. Sci Rep 8:16592. https://doi.org/10.1038/s41598-018-34862-1.

Wei HR, Chen X, Zong XJ, Shu HR, Gao DS, Liu QZ (2015) Comparative transcriptome analysis of genes involved in anthocyanin biosynthesis in the red and yellow fruits of sweet cherry (Prunus avium L.). PLoS One 10:e0121164. https://doi.org/10.1371/journal.pone.0121164. eCollection 2015.

Yu YW, Wang J, Li SH, Kakan X, Zhou Y, Miao YC, Wang FF, Qin H, Huang RF (2019) Ascorbic acid integrates the antagonistic modulation of ethylene and abscisic acid in the accumulation of reactive oxygen species. Plant Physiol 179:1861-1875. https://doi.org/10.1104/pp.18.01250. Epub 2019 Feb 5.

Zeven AC (1991) Wheats with purple and blue grains: a review. Euphytica 56:243-258.

Zhang YZ, Xu SZ, Cheng YW, Ya HY, Han MJ (2016) Transcriptome analysis and anthocyanin-related genes in red leaf lettuce. Genet Mol Res 15. https://doi.org/10.4238/gmr.15017023.

Zhang Z, Guo Y (2018) Hormone treatments in studying leaf senescence. Methods Mol Bio 1744:125132. https://doi.org/10.1007/978-1-4939-7672-0_11.

Zhang ZL, Liu C, Guo YF (2020) Wheat transcription factor TaSNAC11-4B positively regulates leaf senescence through promoting ROS production in transgenic Arabidopsis. Int J Mol Sci 21:7672. https://doi.org/10.3390/ijms21207672.

Zhou XF, Hua DP, Chen ZZ, Zhou ZJ, Gong ZZ (2009) Elongator mediates ABA responses, oxidative stress resistance and anthocyanin biosynthesis in Arabidopsis. Plant J 60:79-90. https://doi.org/10.1111/j.1365-313X.2009.03931.x. Epub 2009 May 23.

\section{Figures}


A

ID

10dpa VS 25dpa 10dpa VS 35dpa 25dpa VS 35dpa

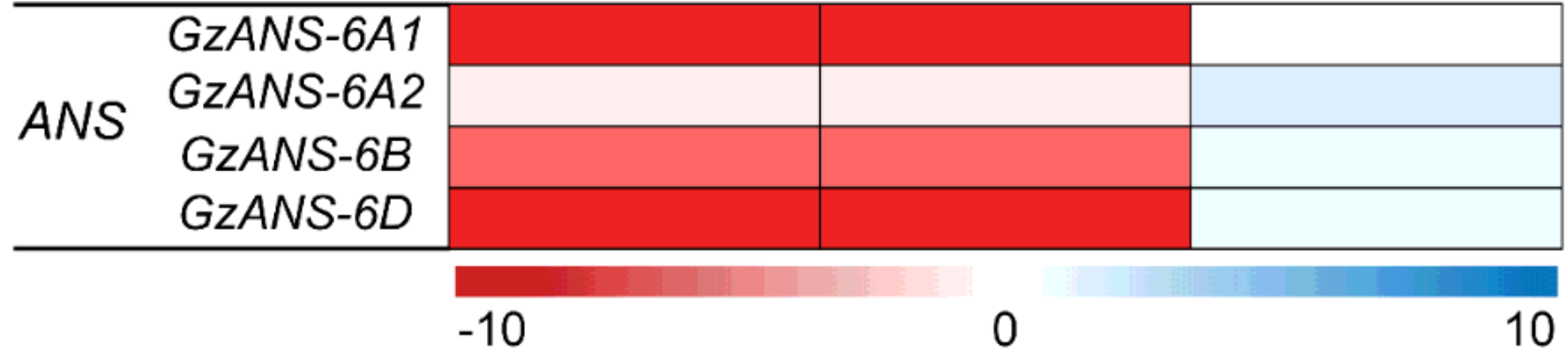

B

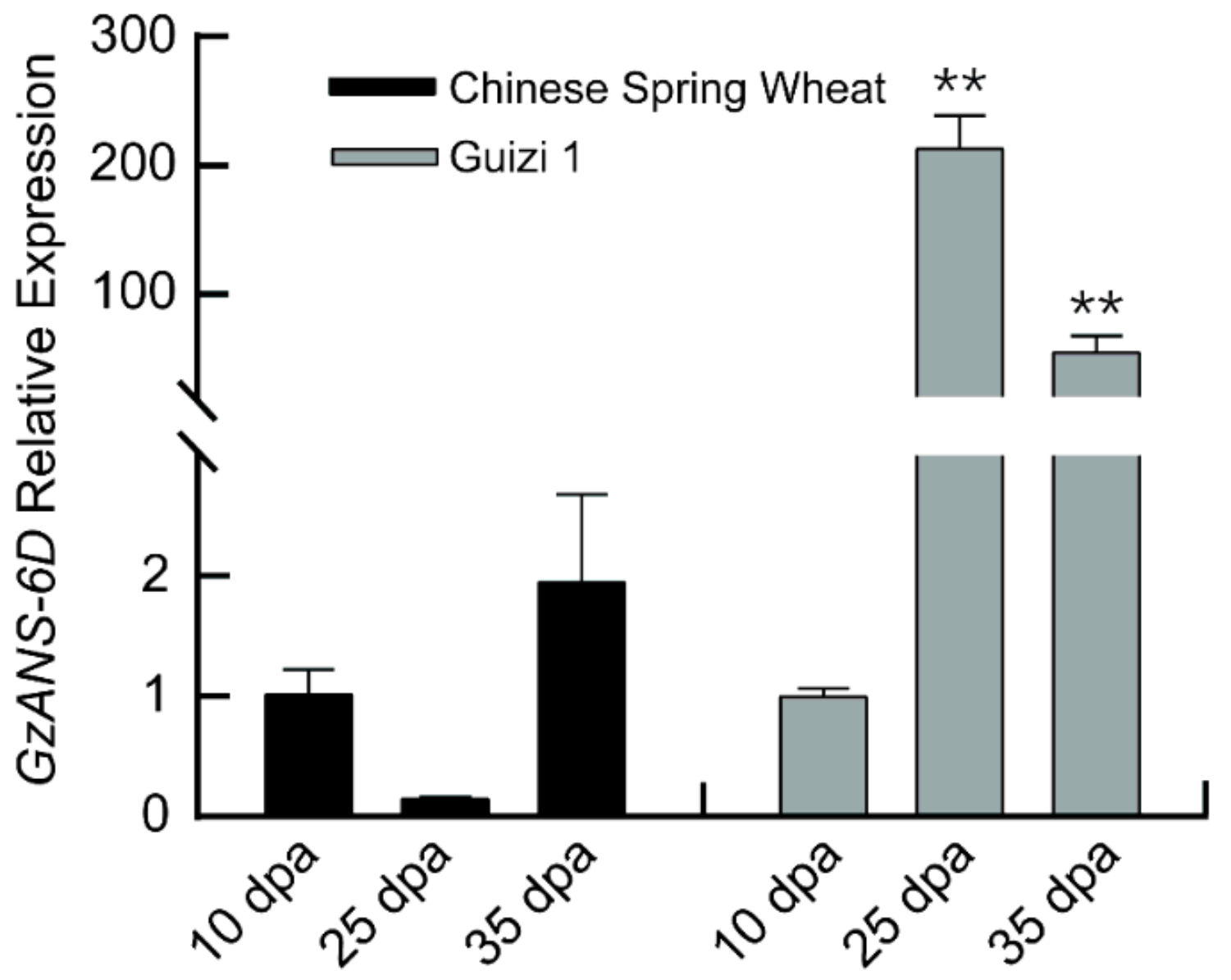

Figure 1

$<\mathrm{p}>$ Heat map and qRT-PCR validation of $<$ em $>$ GzANS $</$ em $>$ (anthocyanidin synthase) expression levels during different wheat developmental periods. (A) Heat map of $<$ em $>$ GzANS $</ e m>$ genes. (B) Expression pattern of $<$ em $>$ GzANS-6D $</$ em $>$ at different grain developmental stages at 10, 20 and $35 \mathrm{dpa}$. Different combinations of numbers and letters (such as 6D, D1, A1, etc.) represent different chromosomal locations. The same chromosome with different numbers indicates corresponding copies of one gene on the same chromosome. Each bar shows the mean \pm SD of triplicate assays. ' $* \star$ ' indicates a significant difference $(<\mathrm{em}>\mathrm{P}</ \mathrm{em}>\& \mid \mathrm{t}$; 0.01$) .</ \mathrm{p}>$ 
Figure 2

$<$ p>Phenotypic observations and anthocyanin and chlorophyll content determinations in WT and transgenic plants. (A) Phenotypic observations of WT and 10-week-old <em $>0 x$ GzANS-6D $</$ em> transgenic lines. (B) Anthocyanin content analysis. (C) Chlorophyll content analysis. Each bar shows the mean \pm SD of triplicate assays. ' $*$ ' indicates a significant difference ( $<$ em $>P</ e m>\& \mid t$; 0.05$)$, and ' $* \star$ ' indicates a highly significant difference $(<\mathrm{em}>\mathrm{P}</ \mathrm{em}>\& \mid \mathrm{lt} ; 0.01) .</ \mathrm{p}>$
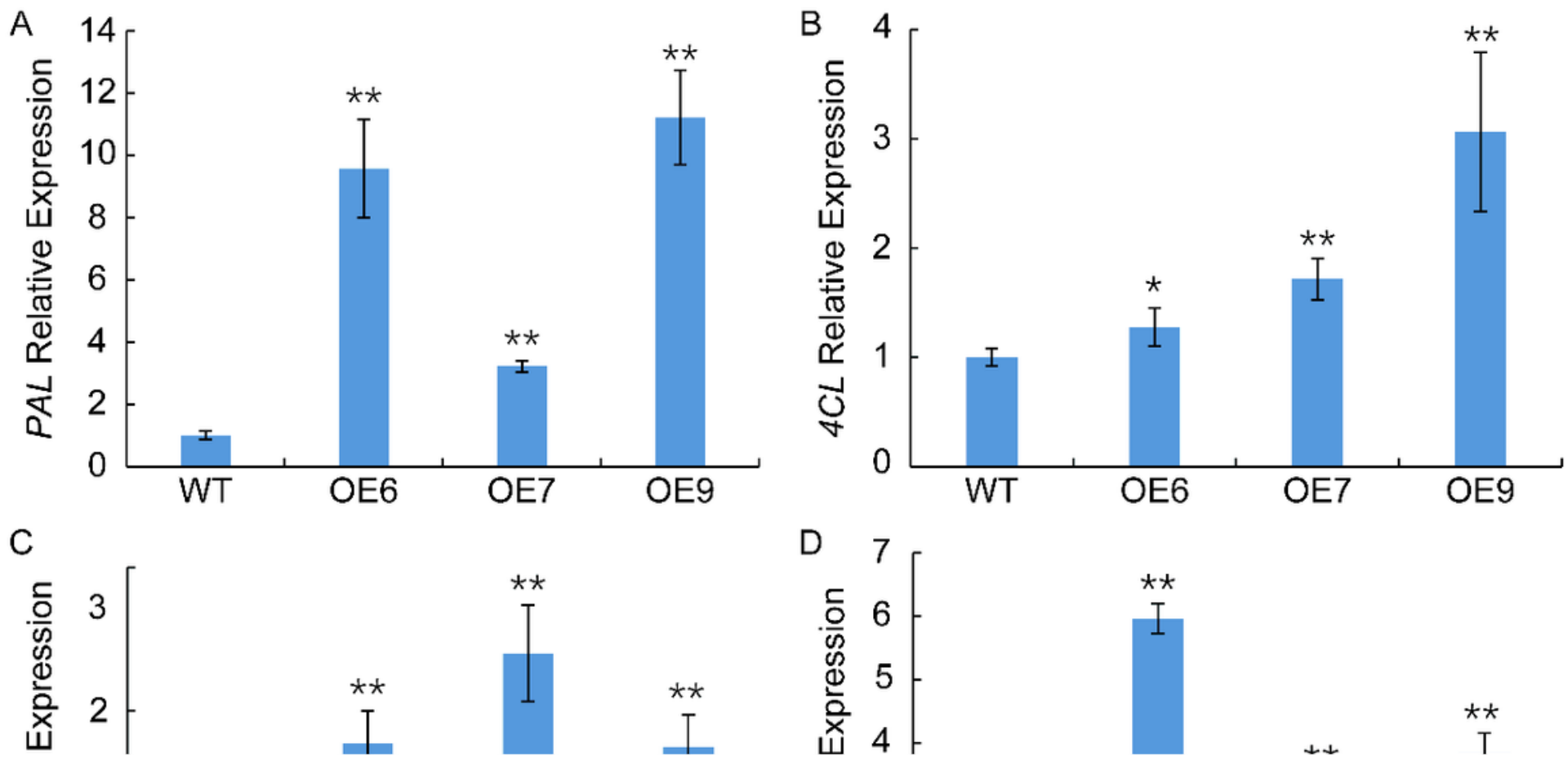

$\star \star$
$\mathrm{T}$ 
$<p>q R T-P C R$ validation of anthocyanin synthesis-related gene expression levels in WT and transgenic plants. (A-F) Expression levels of phenylalanine ammonia-lyase $<$ em $></ e m>(<e m>P A L</ e m>), 4-$ coumarate-CoA ligase $(<\mathrm{em}>4 \mathrm{CL}</ \mathrm{em}>)$, chalcone synthase $<\mathrm{em}></ \mathrm{em}>(<\mathrm{em}>\mathrm{CHS}</ \mathrm{em}>)$, chalcone isomerase $<\mathrm{em}></ \mathrm{em}>(<\mathrm{em}>\mathrm{CHI}</ \mathrm{em}>)$, flavanone 3-hydroxylase $<\mathrm{em}></ \mathrm{em}>(<\mathrm{em}>\mathrm{F} 3 \mathrm{H}</ \mathrm{em}>)$ and dihydroflavonol 4-reductase $<\mathrm{em}></ \mathrm{em}>(<\mathrm{em}>\mathrm{DFR}</ \mathrm{em}>)$, respectively. Each bar shows the mean $\pm \mathrm{SD}$ of triplicate assays. ' $*$ ' indicates a significant difference $(<\mathrm{em}>\mathrm{P}</ \mathrm{em}>\& \mid \mathrm{tt} ; 0.05)$, and ' $\star \star \star$ ' indicates a highly significant difference $(<e m>P</$ em $>\& \mid t ; 0.01) .</ p>$
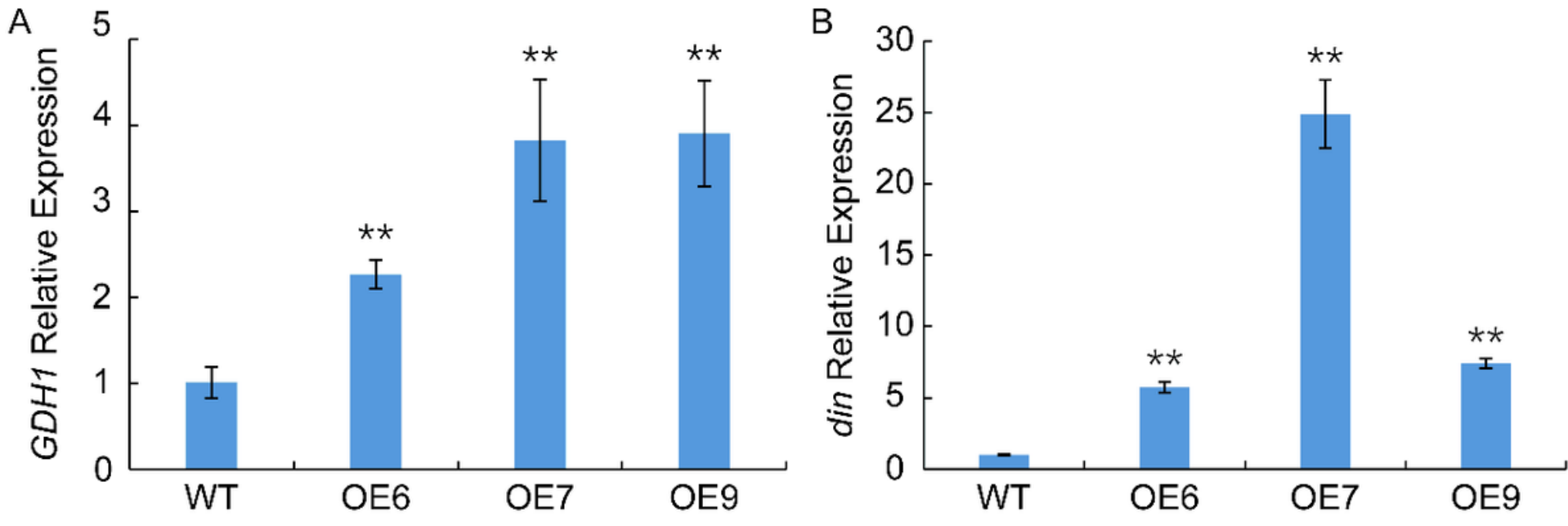

C
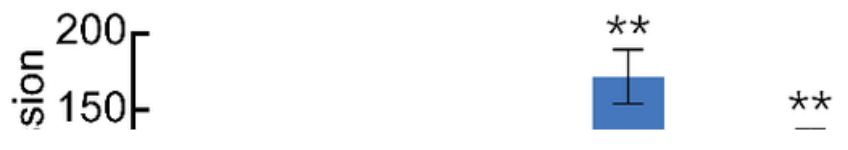

D

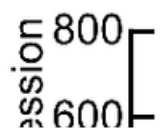

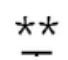

\section{Figure 4}

$<p>q R T-P C R$ validation of senescence-related gene expression levels in WT and transgenic plants. (A-D) Expression levels of $<\mathrm{em}></$ em $>$ glutamate dehydrogenase $<\mathrm{em}></ \mathrm{em}>(<\mathrm{em}>\mathrm{GDH} 1</ \mathrm{em}>)$, $<\mathrm{em}>\mathrm{din}</ \mathrm{em}>$ (a tobacco senescence-associated gene), cysteine protease $<\mathrm{em}></ \mathrm{em}>(<\mathrm{em}>\mathrm{CP} 1</ \mathrm{em}>$ ) and senescence-associated gene ( $<\mathrm{em}>\mathrm{SAG} 12</ \mathrm{em}>$ ), respectively. Each bar shows the mean $\pm \mathrm{SD}$ of triplicate assays. ' $* \star \prime \prime$ indicates a significant difference $(<e m>P</$ em $>\& \mid t ; ; 0.01) .</ p>$

\section{Figure 5}


$<p>$ Analysis of ROS accumulation levels in WT and transgenic plants. (A) Observation of DAB staining for $\mathrm{H}<$ sub $>2</$ sub $>0<$ sub $>2</$ sub $>$. (B) Observation of NBT staining for $0<$ sub $>2</$ sub $><$ sup $>-</$ sup $>$. (C) Calculation of gray values for DAB staining. (D) Calculation of gray values for NBT staining. Each bar shows the mean $\pm S D$ of triplicate assays. ' ${ }^{\prime}$ ' indicates a significant difference (P \& lt; 0.05), and ' $* *$ ' indicates a highly significant difference $(<e m>P</ e m>\& \mid t ; 0.01) .</ p>$

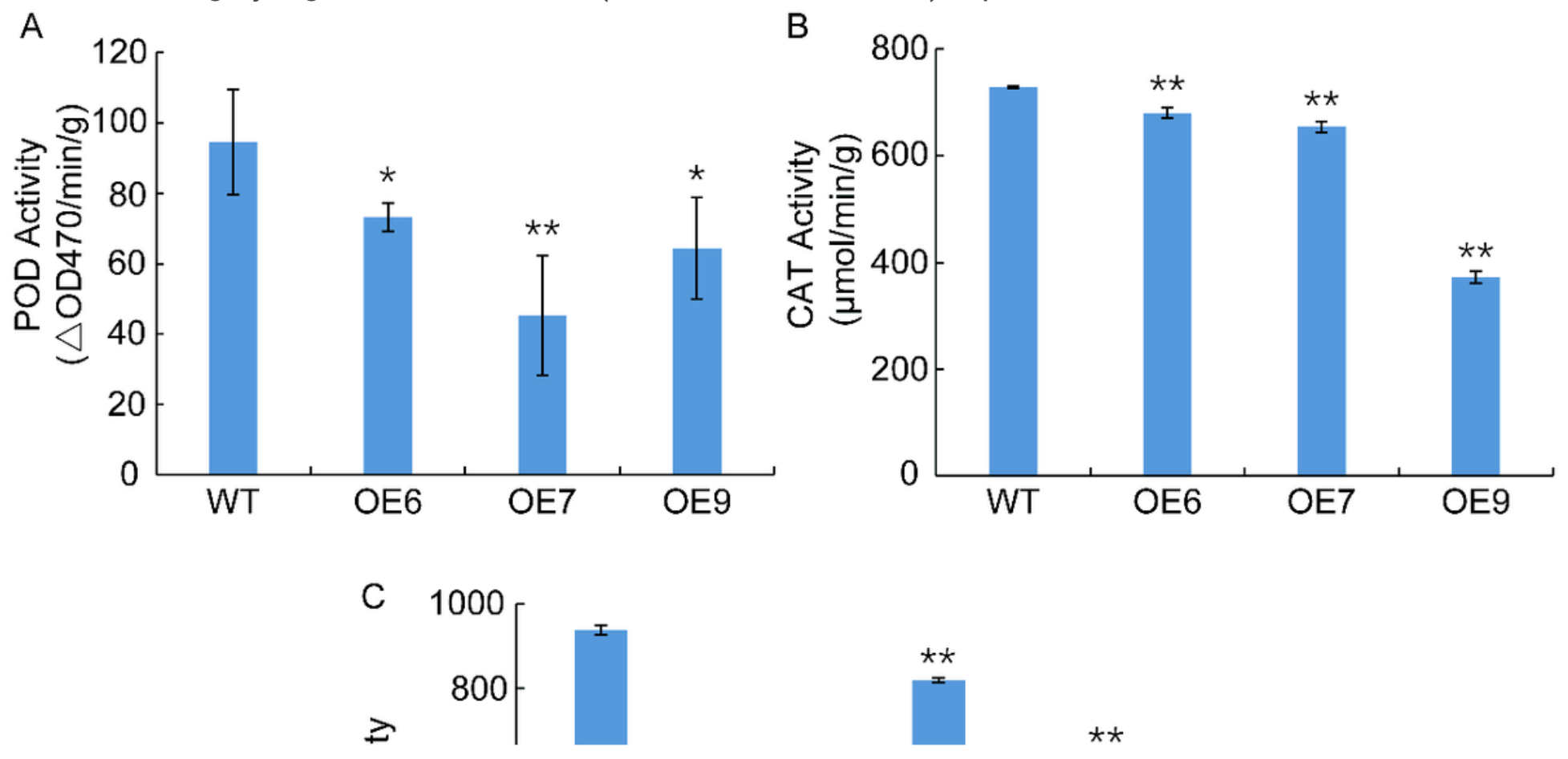

Figure 6

$<p>$ Activity levels of antioxidant enzymes in WT and transgenic plants. (A-C) Activity levels of POD, CAT and SOD, respectively. Each bar shows the mean \pm SD of triplicate assays. ' $*$ ' indicates a significant difference (<em $>\mathrm{P}</ \mathrm{em}>\& \mid \mathrm{t}$; 0.05), and ' $*{ }^{\prime}$ ' indicates a highly significant difference $(<\mathrm{em}>\mathrm{P}</ \mathrm{em}>\& \mid \mathrm{lt}$; $0.01) .</ p>$ 
A

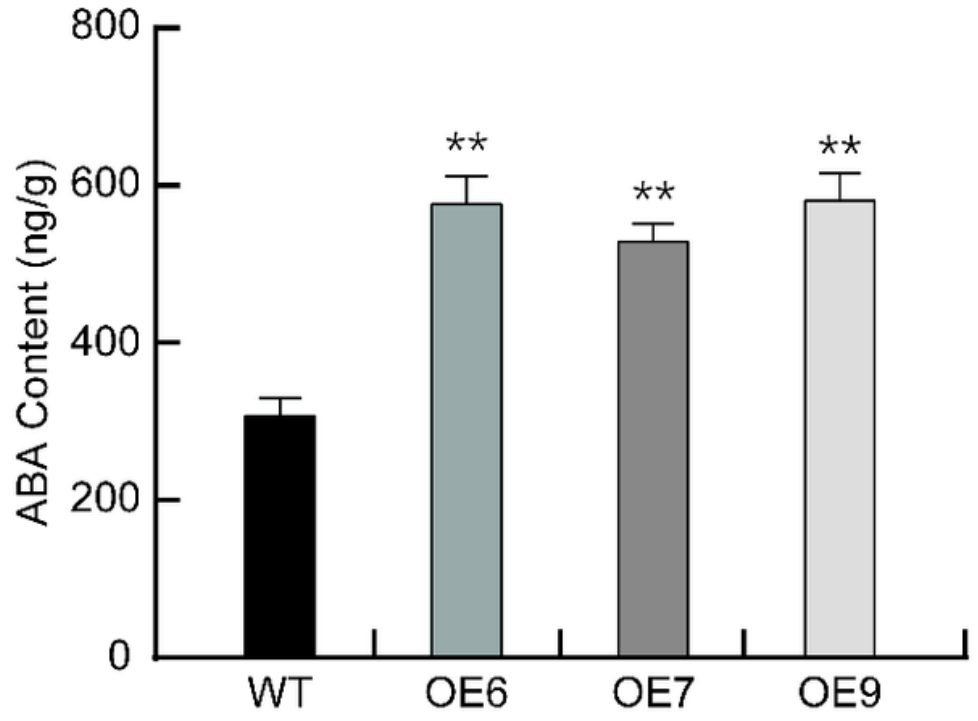

B

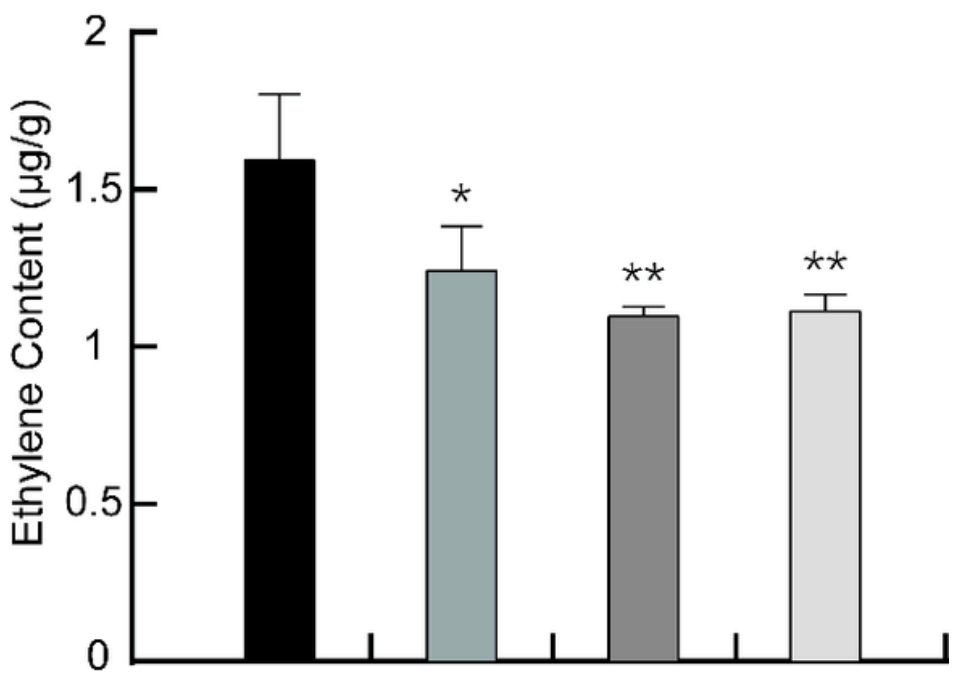

Figure 7

$<p>A B A$ and ethylene contents in WT and transgenic plants. A) Analysis of ABA content. B) Analysis of ethylene content. Each bar shows the mean $\pm S D$ of triplicate assays. The ' $*$ ' represents significant differences (<em $>\mathrm{P}</ \mathrm{em}>\& \mathrm{lt}$; 0.05$)$ and the ' ${ }^{\prime \prime}$ ' represents significant differences ( $\left.<\mathrm{em}>\mathrm{P}</ \mathrm{em}>\& \mathrm{lt} ; 0.01\right)$. $</ p>$

\section{Figure 8}

$<p>$ The qRT-PCR validation of chlorophyll degradation-related genes and ABA-related genes in WT and transgenic plants. ( $A$ and $B$ ) Expression levels of ABRE-binding transcription factors $<e m>A B F 1</ e m>$ and $<\mathrm{em}>\mathrm{ABF} 2</ \mathrm{em}>$, respectively. (C-F) Expression levels of pheophorbide a oxygenase (<em $>\mathrm{PAO}</$ em $>$ ), non-yellow coloring $<$ em $></$ em $>(<e m>\mathrm{NYC}</ \mathrm{em}>)$, non-yellowing $(<\mathrm{em}>\mathrm{SGR}</ \mathrm{em}>$, also known as $<$ em $>\mathrm{NYE}</ \mathrm{em}>$ ) and chlorophyllase $<\mathrm{em}></ \mathrm{em}>(<\mathrm{em}>\mathrm{CHL}</ \mathrm{em}>)$, respectively. Each bar shows the mean \pm SD of triplicate assays. ' $* \star$ ' indicates a significant difference $(<e m>P</ e m>\& \mid t ; 0.01)$. $</ p>$ 

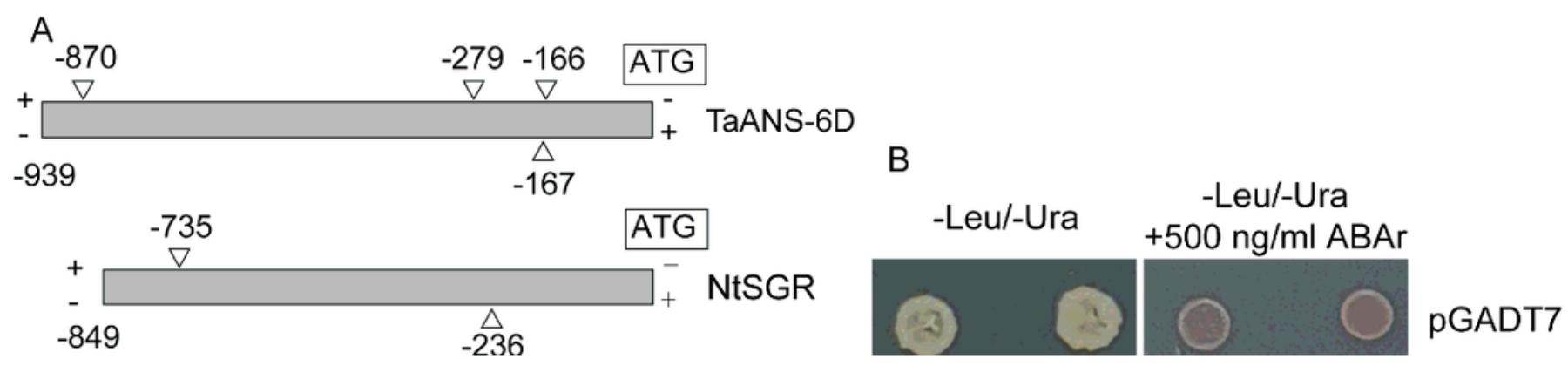

\section{Figure 9}

$<p>Y 1 H$ assay. (A) Schematic diagrams of ABRE elements identified in the promoter regions from $<\mathrm{em}>\mathrm{GzANS}-6 \mathrm{D}</ \mathrm{em}>$ and $<\mathrm{em}>\mathrm{NtSGR}</ \mathrm{em}>$. Triangles, ABREelements; numbers, distance (bp) from ATG; +, distance from ATG in a positive DNA strand; -, distance from ATG in a negative DNA strand; grey portion, the region cloned into Bait vector in $\mathrm{Y} 1 \mathrm{H}$ assay. (B) $\mathrm{Y} 1 \mathrm{H}$ assay analyze that NtABF1 and GzABF1 interacted with the promoter of $<$ em $>$ GzANS-6D $</ e m>$. (C) $Y 1 H$ assay analyze that NtABF2 interacted with the promoter of $<\mathrm{em}>\mathrm{NtSGR}</ \mathrm{em}>.</ \mathrm{p}>$

\section{Supplementary Files}

This is a list of supplementary files associated with this preprint. Click to download.

- SupplementaryMaterial.docx 